\title{
Black women's hair: the main scalp dermatoses and aesthetic practices in women of African ethnicity*
}

\author{
Aline Tanus ${ }^{1}$ \\ Delky Johanna Villarreal Villarreal ${ }^{1}$ \\ Maria Fernanda Reis Gavazzoni Dias ${ }^{1,2}$
}

\author{
Camila Caberlon Cruz Oliveira ${ }^{1}$ \\ Fernando Andres Vargas Sanchez ${ }^{1}$
}

DOI: http:/ / dx.doi.org/10.1590/abd1806-4841.20152845

\begin{abstract}
Afro-ethnic hair is different from Caucasian and Asian hair and has unique features. Ethnic hair is more prone to certain conditions or diseases. Such diseases are not only related to the fragile inner structure of the hair, but also to the cultural habits of hairstyles that often exert traction forces upon the pilosebaceous follicle. Women with African hair subject their hair to chemical treatments such as hair straightening and relaxing, and thus modify the structure of their hair shaft, making it more susceptible to damage. For this reason, hair complaints are common among black women and represent a diagnostic challenge to the dermatologist, requiring a thorough clinical examination of the hair and scalp, and a detailed medical history of the patient. The purpose of this review is to warn of the potential side effects and sequelae related to hairstyles and hair treatments used by black women, and to highlight the major diseases that affect this ethnicity.
\end{abstract}

Keywords: African continental ancestry group; Alopecia; Esthetics; Hair diseases; Scalp dermatoses; Women

\section{INTRODUCTION}

The appearance and aesthetics of the hair are a frequent concern of women around the world, who increasingly try to follow the trend of straightening their hair. The habit of straightening Afro-ethnic hair probably started during the slavery period, when black women began to use hot irons to straighten their hair. Hair-straightening techniques have been improved ever since, starting with the hot comb - created by Madame CJ Walker in the twentieth century -, and being followed by the development of chemical hairstraightening products.

This review aims to describe the most common hairstyles and the main scalp dermatoses of women of African descent. Moreover, we try to describe the unique characteristics that contribute to the development of specific diseases in this important ethnic group in a miscegenated country like Brazil.

\section{THEAROETHNICHAIR}

Although there are numerous studies on human hair, there are only a few publications about the influence of hair care practices and cultural habits on African hair and scalp health and conditions. ${ }^{1,2}$ The term race or ethnicity applies to groups of people who have specific similar features, such as skin color, hair type, cranial and facial conformation, and

Approved by the Advisory Board and accepted for publication on 01.08.2014. 
genetic ancestry, which are the remainings of the first adaptations to temperature and other environmental influences over several generations. ${ }^{3}$ For classification purposes, the hair can be divided into three main groups according to their racial origin: Caucasian, Oriental and African. These differ mainly in their appearance, geometry, mechanical properties and water content. ${ }^{1}$ These differences in the African hair explain why some types of cicatricial alopecia and hair diseases are more prevalent in or almost exclusive to this ethnic group. ${ }^{1,3}$

Granular pigments (eumelanin), which vary in color from black to dark red, give darker colors to the hair, while diffuse pigments (pheomelanin), which vary in color from bright red to pale yellow, give lighter colors to the hair. Afro-ethnic hair characteristically contains more eumelanin than pheomelanin and is therefore darker. ${ }^{4}$

The hair follicles are asymmetrical, with elliptical or oval cross section and curve hair follicle bulb. This elliptical shape with flattened and irregular hair shafts is responsible for lower resistance and a higher susceptibilityto breakage when compared with Caucasian and Asian hair, which have a straight hair follicle, free of irregularities, and round-shaped hair shafts with homogeneous diameter throughout the fiber. ${ }^{3,5,6,7}$

Because of this peculiar anatomical aspect, scalp biopsies should be carried out with care, respecting the inclined axis of implantation of the hair shaft. Thus, the biopsy must be performed parallel to the axis of growth of the hair, which in the case of people of African descent, has a 45 degrees tilt angle. Therefore, the vertical section of the test must be obtained by inserting the punch (of at least $4 \mathrm{~mm}$ in size) at 45 degrees in relation to the skin, reaching subcutaneous tissue. ${ }^{8}$ The horizontal section does not suffer changes in comparison to other ethnic groups: the fragment is collected parallel to the epidermis, below the dermoepidermal junction $(0.5$ to $1 \mathrm{~mm}){ }^{4}$ For an adequate evaluation procedure, two biopsy samples should be taken from representative pathology sites. The ideal fragment is taken from the place where there is a positive traction test, representing active disease. ${ }^{9}$

The water absorption capacity and the bonds between the water molecules and the protein molecules of the shaft interfere with the physical properties and the architecture of the hair. Both of them are reduced in the Afro-ethnic hair and, for that reason, the hair strands form knots and longitudinal cracks that contribute even more to the relative fragility of this hair type.,10

Black women have shorter hair when compared with other types of hair, even after a prolonged period without cutting. This is explained by the fact that Afroethnic hair grows more slowly than Caucasian hair (0.9 $\mathrm{cm} /$ month and $1.3 \mathrm{~cm} /$ month, respectively)., ${ }^{2,3}$ In his book, The hair in black women, Dr. Neil Persadsingh compared African, Asian and Caucasian hairs removed while combing and concluded that African hairs have a higher number of knots, partial breakages, trichoptilosis (longitudinal division of the distal shaft) and breakages. ${ }^{10}$ In addition, the hair density and the total number of terminal follicles are smaller when compared to Caucasians. However, the fibers have a similar chemical composition in terms of proteins and amino acids. ${ }^{2,3}$

Sebum secretion is normal, reduced or increased in the scalp of individuals of African descent. Nevertheless, the curled confi guration of the shaft impairs the normal distribution of natural oils along the hair shaft, from root to tip, providing a dull and dry look. In straight Asian and Caucasian hair, the straight and regular surface allows a better reflection of light and a homogeneous distribution of sebum over the hair strands, making them look brighter and more moisturized. ${ }^{6,7}$

Although African hair is drier more brittle and susceptible to chemical and physical damage due to its spiral structure, it also has some advantages, especially in hot climates where it forms a natural barrier against the sun. Its spiral curved form causes the air to cool up and easily circulate through the scalp, being part of the body's thermoregulatory mechanism. Another fact which constitutes a thermoregulatory advantage is that fluids like water and sebum do not distribute themselves completely evenly along the hair strands, reducing the water loss capacity and further contributing to the regulation of body temperature. ${ }^{10}$

\section{ANAMNESISINBLACKWOMEN}

When investigating hair loss, the following questions should be asked ${ }^{11}$ :

- When was the onset of symptoms?

- Did the hair loss begin centrally and then spread symmetrically in all directions?

- Was there erythema, scaling, itching or burning sensation?

- Does the hair loss continue progressing or has become stable?

- Did the patient make use of chemical products such as chemical straighteners, permanent wavers or relaxers?

If the clinical examination reveals fracture of the hair shaft it is imperative that the physician ask the patient whether she has used chemical relaxers such as sodium hydroxide, guanidinium hydroxide, and ammonium thioglycolate (which can be used for both permanent hair waving and for hair straightening) 
or chemical hair straighteners with formaldehyde or formaldehyde releasers, such as glyoxylic acid and methyleneglycol. Completely broken hair shafts are a very common result of the use of different types of mutually incompatible chemical straighteners. Thioglycolate, for instance, is incompatible with hydroxides. The use of both substances on the same site causes immediate hair loss due to complete breakage of the hair shaft. Other causes of hair shaft breakage are the frequent use of hair-straightening and hair-waving irons, or the habit of combing the hair using a hair comb made of plastic or other good electricity-conducting material. ${ }^{11}$

\section{CINICAL EXAMINATION}

Clinical examination should include a direct questioning about the use of hairpieces or braids, which constitute important behavioral and cultural factors involved in some cases of alopecia. It is important to evaluate the situation of the hair shaft and the hair follicle by means of a traction test and of dermoscopy. The pattern of hair loss and the scalp should also be evaluated in order to determine whether there is hair breakage or the presence of hairstyles or hairpieces that apply traction to the follicles. ${ }^{10,11}$

\section{AFRICAN SCALP DERMATOSES}

\section{a. Hair Breakage: acquired proximal} trichorrhexis nodosa (APTN)

In this entity, the hair is so fragile that a minor trauma to its proximal end causes its breakage. In areas damaged by the act of combing the hair or applying pressure to it (hairstyles or positioning of the side of the head on the pillow during sleep) this usually gives the appearance that the hair was cut very close to the scalp. ${ }^{11}$

The disease affects patients of all ages and both sexes, and may be associated with a family history of "hair that does not grow." Although some patients have a congenital defect in the formation of keratin, trichorrhexis nodosa is more commonly acquired, resulting from physical or chemical trauma. ${ }^{12}$

The onset of APTN may occur after years of use of chemicals or heat devices for straightening the hair. The continuous and uninterrupted use of chemical straighteners such as sodium hydroxide and guanidine hydroxide weakens the hair shaft by causing the loss of protein material, serious damage to the cuticle and, subsequently, to the cortex. A change in the choice of chemical straightener may result in the simultaneous use of mutually incompatible chemicals and lead to the total breakage of the hair shaft. In some cases, this can result from the excessive combing or brushing of the hair with a very stiff brush and plastic combs. In other cases, it is not possible to correlate the chemical, thermal or mechanical trauma with the development of the disease. It is then believed that these factors act as adjuvants and are influenced by genetic factors inherent to each patient. ${ }^{11}$

Callender VD et al. believe that acquired proximal trichorrhexis nodosa may bean early indicator of central centrifugal scarring alopecia (CCCA). APTN begins with thinning hair on the vertex accompanied by little or no clinical evidence of scarring. During the evolutionary process of normal hair, the cells of the internal follicular sheath become keratinized earlier than the cortex and act as a cylindrical axis that supports the hair growth, which will be guided and shaped by this structure. The reason why some patients with APTN develop CCCA is still unknown. However, it is believed that the absence of internal follicular sheath causes a disorganized 'packaging' of the hair, especially of the cuticle, resulting in instability and ultimately in breakage. ${ }^{13}$

The diagnosis can be made by means of the tug test, which consists in selecting a sample of 10-20 hair strands and holding it at its base with the forefinger and thumb of one hand while the other hand pulls the strands towards their distal end. With this maneuver, it is possible to observe the breakage of the hair in half. and formation of longitudinal cracks along the hair shaft at dermoscopy.11 Small swelling areas or thinning of the cuticle can be observed at the shaft and correspond to the sites that are more susceptible to trauma (trichorrhexis nodosa). Another characteristic feature of the disease is trichonodosis, i.e., the spontaneous formation of knots in the follicular shaft, often seen in very curly hair, probably resulting from the use of hair relaxers and from physical trauma. ${ }^{10,11}$

Recommendations for these patients are: ${ }^{11,12}$

- Avoid the use of hair relaxers and hairstyles; if necessary, use once every 8-12 weeks.

- Avoid the use of physical hair straightening techniques, such as flat irons and hair dryers.

- Avoid excessive combing of the hair. Use a soft brush with widely spaced bristles and coated tips or a wooden comb with wide teeth.

- Avoid massaging or rubbing the hair (scratching due to seborrheic dermatitis has also been described as a trigger for hair breakage).

Even after the complete interruption of all hair straightening and relaxing tecnhiques, it is expected that the disease will remain active for further 2-4 years, possibly because all hairs have to be replaced by new anagens ones. Alternatively, some women choose to keep their hair short in order to achieve that the fractured hairs and the hairs that were not affected by the disease have the same length. ${ }^{11}$

We emphasize the importance of making the 
differential diagnosis between acquired proximal trichorrhexis nodosa and early-onset CCCA, because both conditions have the same topography and do not present obvious signs of a scarring area, especially in women of African descent. In the case CCCA, the simple recommendation to interrupt all hair treatments will be ineffective. Here is also necessary the introduction of treatment in order to cease the progression of the disease, reduce the chances of developing scarring alopecia and increase the probability of hair regrowth in the future. ${ }^{13}$

\section{b. Short Anagen Syndrome}

This syndrome is characterized by the inability of the hair to grow long and the observation of an increased number of telogen hairs in the trichogram. Usually affected are Caucasian children with blond thin hair who are brought to the doctor by their parents because their hair does not grow long or because they have never had a haircut. However, Nidhi Avashia et al. reported a case of short anagen syndrome in a 38-year-old black women whose main complaint was that she had had short hair since her childhood. The patient also reported that her first haircut had occurred when she was seven years old and that her hair had never grown more than $7.6 \mathrm{~cm} .{ }^{14}$

The diagnosis is made by means of the observation of the presence of normal hair, with a short anagen phase and there may be an anagen to telogen ratio of up to $62: 38 . .^{14}$

It is important to keep in mind that this is a possible diagnosis in black children with limited growth of hair. Ignorance of this entity may lead to a diagnosis of traction alopecia in children who wear braids and do not present normal hair growth. The main differential diagnosis is the loose anagen syndrome, characterized by the presence of more than $70 \%$ of anagen hairs and usually no telogen hairs in the trichogram. Short anagens were also described in the tricho-dental syndrome, a congenital disorder associated with hypodontia. ${ }^{15}$

Therapeutic options are limited. Studies describe the use of minoxidil $5 \%$ to prolong the duration of the anagen phase. ${ }^{16}$

\section{c. Traction alopecia}

Traction alopecia (TA) is an extremely common condition in black women, resulting from years of use of hairpieces and hairstyles that exert prolonged and repeated traction upon the hairs and cause the shortening of the hairs in the frontotemporal line of implantation of the hair. ${ }^{11,17}$

Usually, the areas of alopecia are symmetrical along the frontotemporal line (Figure 1). ${ }^{12}$ The involvement of the occipital region is unusual. ${ }^{12}$ The earliest sign of TA is the perifollicular erythema,

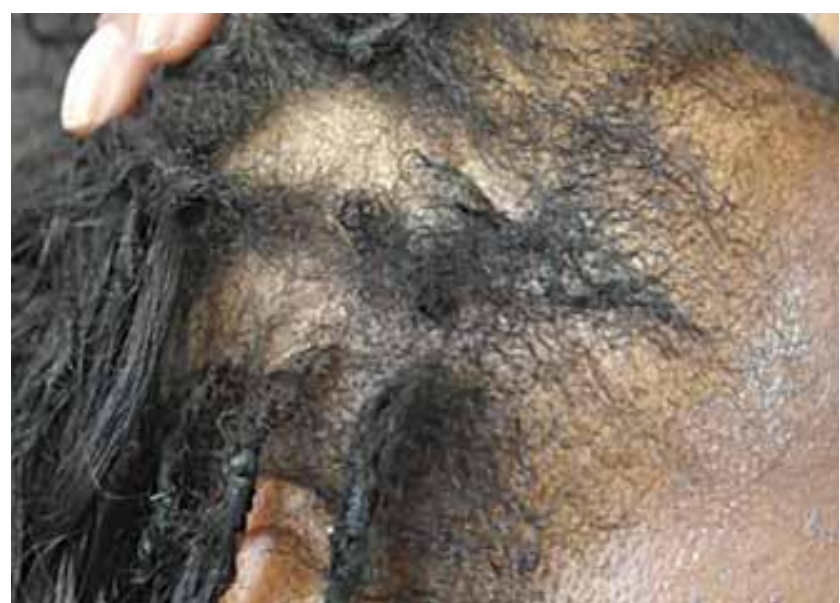

Figure 1: Traction alopecia. Observe the traction exerted by the hairpiece upon the hairs, resulting in the shortening of the hairs in the temporal region

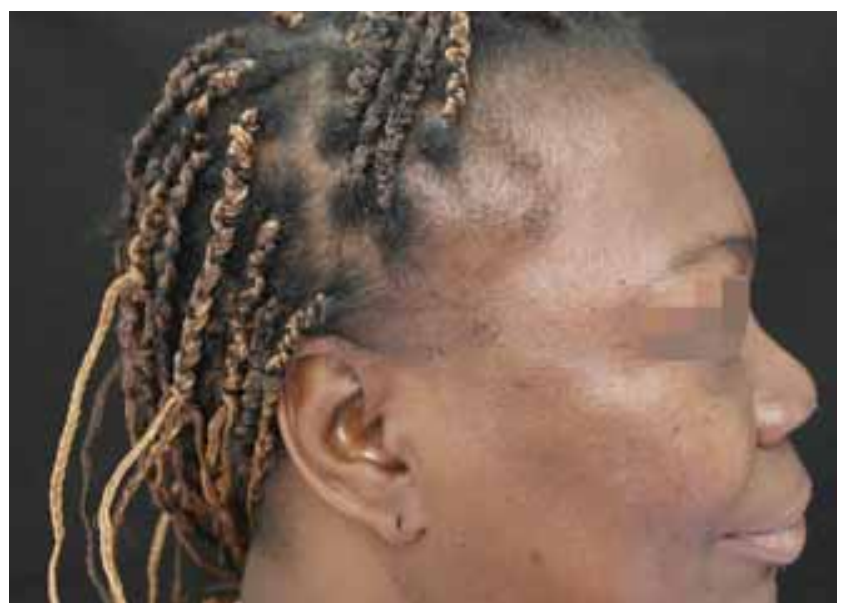

FiguRE 2: The fringe sign: short tonsured hairs outlining the original frontotemporal line of implantation of the hair

which may progress to folliculitis if the trauma is continuous., ${ }^{4,17,18}$

The presence of short hairs scattered along the frontotemporal line is a characteristic finding of TA and is called "fringe sign" (Figure 2). ${ }^{17}$ The the pathogenesis of TA remains unknown, however, it seems to start gradually with the shortening of marginal hairs, suggesting that it induces follicular miniaturization. ${ }^{19,20}$ It is assumed that the hairs localized placed implanted along the margins are shorter, and therefore become detached from the tight ponytail, thereby giving the characteristic feature of fringe sign. ${ }^{17}$ 
In patients who have no clinical history of use of tight hairstyles, the differential diagnosis is broad and includes androgenetic alopecia, telogen effluvium, trichotillomania, primary lymphocytic scarring alopecias (lichen planus pilaris, central centrifugal scarring alopecia, Brocq's pseudopelade and frontal fibrosing alopecia ), and alopecia areata. ${ }^{17}$

Less often, TA may affect the occipital region. In this case, the differential diagnosis with ophiasis alopecia areata is imperative.

Alopecia areata (AA) is a type of non-scarring alopecia of autoimmune etiology. Dermoscopic findings of AA are the unique exclamation mark hairs or pencil hairs. Histopathologic examination shows lymphocytic infiltrates around the follicular bulge.

Ophiasis AA may be underdiagnosed in black women, since there is a tendency to diagnose the condition in this population as TA. Therefore, it is important to investigate the history of use or traumatic removal of hairpieces, which speaks for the diagnosis of traction alopecia..$^{21,22}$

Studies with African women have shown as risk factors for the development of TA: ${ }^{23}$

1- traction exerted upon chemically treated hair. Hairstyles should be done at least two weeks after the chemical treatment and hair relaxers should be avoided, especially in children. If a chemical hair relaxer is used, it should only be applied to the virgin hair, and one should be careful not to apply it to previously relaxed hair.

2- women who complained of pain, tingling, papules or crusts.

3- advanced age, probably due to the chronic use of hairstyles that damage the hair.

4- combined use of hair coloring products and relaxers (should be done at least two weeks apart)

5- thermal injury on relaxed hair. The use of hair dryers and flat irons should be avoided.

In the early stages, TA presents trichomalacia and an increased number of telogen and catagen hairs. In the course of the disease, a phenomenon similar to a "follicular abandonment" occurs: the terminal hairs disappear from the follicles, but the vellus hairs remain intact. In advanced disease settings, there may be a decrease of sebaceous glands, a mild inflammatory infiltrate and a decrease in the number of terminal follicles, which are replaced by fibrotic tissue. ${ }^{4,17,24}$

Dermoscopy shows many miniaturized hairs and pinpoint white dots (visible acrosyringium). Due to the traction, the hairs may also show signs of breakage of the follicular shaft, and sometimes display cylinders of the follicular sheath - which was freshly detached due to the trauma (hair casts)-, as well as cadaveric hairs and longitudinal slits at the distal end

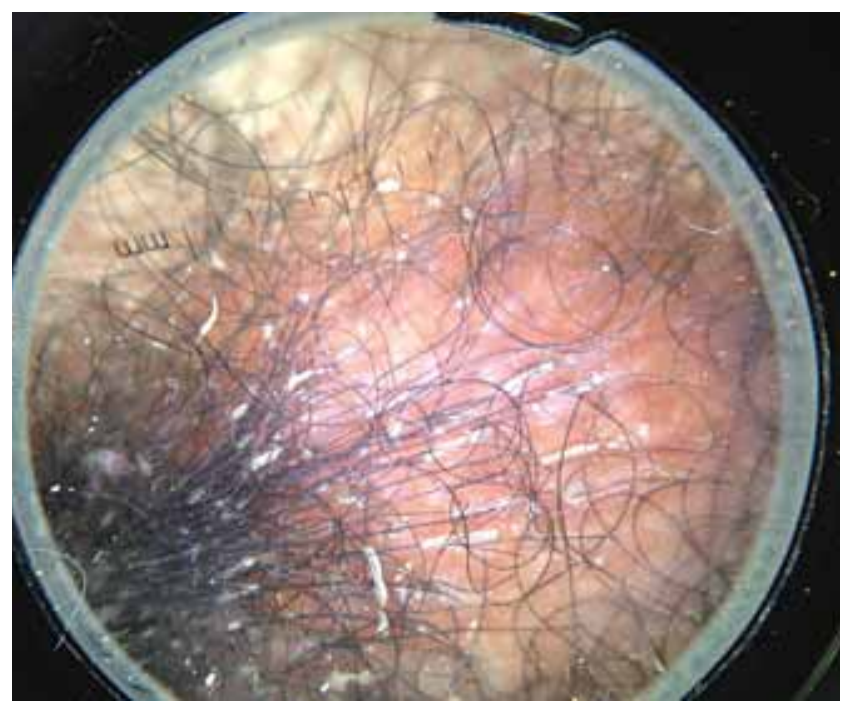

Figure 3: Observe the hair casts in traction alopecia, representing follicular sheath cylinders which detach themselves due to the traction forces exerted upon the hairs and adhere themselves to the hairs

of the hair shaft (Figure 3) .

The prognosis is variable. If the diagnosis is made early and the use of hairstyles and hair techniques that exert traction upon the hairs is stopped early (in childhood), the clinical status may be reversible, usually with full recovery and growth of new hair. However, in the case of a late diagnosis, the chronic perifollicular inflammation may lead to the formation of fibrotic scarring tissue and the alopecia may become permanent. ${ }^{11}$

Thus, treatment will depend on the stage of the disease. In childhood, the most important action is prevention. Patients and their parents should be instructed to avoid using tight hairstyles that exert too much traction upon the hair and scalp..$^{10,11}$

In the early stages of TA it is also important to avoid using chemicals and thermal sources for hair straightening/relaxing, as well as the excessive combing of the hair, because hair loss is still reversible at these stages. ${ }^{17}$ The application of intralesional corticosteroids may be useful for reducing the perifollicular inflammation in adults with early-stage TA. ${ }^{12,17}$ Topical and oral antibiotics are also reported as therapeutic options in cases of folliculitis. Minoxidil $2 \%$ and $5 \%$ are used in some studies to stimulate hair regrowth. ${ }^{17,25}$

In the advanced stages of TA surgical treatment should be considered. Micro-implants, mini implants, follicular unit transplantation and rotation flaps have 
been described as effective treatments. ${ }^{12,26}$

\section{d. Scarring alopecias}

Scarring alopecias represent a group of disorders whose common final pathway culminates in the destruction of the hair follicle and its replacement by fibrous tissue. The alopecia may occur as a primary event when the follicle is the main target of the pathological process (primary scarring alopecia) or as a secondary event when the follicle acts as an "innocent bystander" in the course of a disease, which occurs outside the follicular unit (secondary scarring alopecia). ${ }^{27}$

In 2001, the North American Hair Research Society (NAHRS) proposed a classification for primary scarring alopecias, based on the predominance of inflammatory cells in the hair follicle. Four groups were considered: alopecias with predominantly lymphocytic infiltrate, with predominantly neutrophilic infiltrate, with mixed infiltrate and with nonspecific infiltrate. ${ }^{28}$

Below is a brief review of the major primary scarring alopecias in women of African descent.

\section{d.1) Central Centrifugal Cicatricial Alopecia}

Central centrifugal cicatricial alopecia (CCCA) is defined as a hair loss that starts at the vertex and middle region of the scalp and progresses centrifugally. It is the most common form of primary scarring alopecia in people of African descent, affecting more women than men. The prevalence increases with age and the disease is more common at the end of the second and in the third decades of life..$^{22,29}$

In 1968, LoPresti and colleagues first used the term "hot comb alopecia" to describe a variant of scarring alopecia that was associated with the use of hot metal combs by African-American women. It was believed that the hot Vaseline used in some hair treatments caused chronic inflammation of the hair follicle, with its subsequent destruction and replacement with fibrous tissue. The cause of this type of scarring alopecia was later discussed. ${ }^{22,30}$

In 1992, Sperling and Sau conducted a retrospective study with 10 women diagnosed with "hot comb alopecia". They found a poor correlation between the use of hot combs and the onset and progression of the disease in these patients. ${ }^{30,31}$

The term "hot comb alopecia" was replaced by the term 'follicular degeneration syndrome', based on histological findings of the disease and on the conclusion of Sperling's research. However, it is now known that this disease has a multifactorial origin, involving intrinsic and extrinsic factors. ${ }^{29}$ For this reason, in 2001, the NAHRS Cicatricial Alopecia Center suggested the denomination 'Central Centrifugal Cicatricial Alopecia' (CCCA), a more descriptive term which encompasses the Follicular Degeneration Syndrome, "hot comb alopecia" and pseudopelade. ${ }^{30}$ All CCCA variants have the following characteristics in common:

(1) chronic and progressive disease, with eventual spontaneous reduction of the inflammation after years or decades

(2) disease predominantly centered on the crown or vertex of the scalp

(3) progresses in a symmetrical way. Disease activity occurs mainly in the peripheral zone, with variable width, surrounding the central zone of alopecia.

CCCA may be classified as 'early stage' (inflammatory) or 'late stage' (cicatricial). ${ }^{22}$ The hair loss starts at the crown or vertex of the scalp and gradually progresses centrifugally. As the disease progresses, it leaves an area of alopecia with irreversible loss of follicular ostia. A few isolated hairs, some of them are short and brittle or present polytrichia, may remain in the central hairless area. ${ }^{4,22}$ CCCA is usually asymptomatic, but may be accompanied by mild symptoms such as pain, itching and/or burning sensation in the scalp. Signs of inflammation are absent, although erythema, follicular pustules, scaling and inflammatory borders in areas of alopecia may arise at any stage of the disease. ${ }^{22,32} \mathrm{~A}$ recent observational study concluded that the breakage of hair in the vertex region may be an early indicator of CCCA. ${ }^{33}$

To date, the etiology of CCCA is controversial. The most likely theory is that it has a multifactorial origin, being implicated as possible predisposing factors: genetics, hair straightening chemicals, trauma caused by traction and the spiral configuration of the hairs. In several studies, the hair care habits of AfricanAmerican women have been associated with the development of CCCA. Irregularities in the diameter along the hair shaft, a thinner thickness, more curly and flattened, and spiral shape of the hairs make Afroethnic hair more susceptible to breakage caused by the hair treatments often used by this population. ${ }^{28,29,34}$

A recent study found that patients who had advanced and severe CCCA used more hairstyles with traction, such as hair braiding, for a long time, which causes chronic folliculitis of the scalp with subsequent hair loss and development of CCCA. The cause and effect relationship of chemical relaxers is still controversial. However, it is known that chemical relaxers weaken the hair shaft, increasing the possibility of hair breakage, and may cause chemical burns on the scalp, although it is not yet proven that they contribute to the development of CCCA., ${ }^{4,34}$ 
Biopsy is important to confirm the diagnosis. Ideally, two fragments should be obtained (for horizontal and vertical histopathological sections) with 4-mm punch biopsy specimens taken from the periphery or the active border of the alopecia area. ${ }^{32}$ The histological findings are nonspecific and may be found in other forms of inflammatory scarring alopecia, however, the premature desquamation of the inner root sheath is the most specific and earliest feature of CCCA. ${ }^{36}$ The involved follicles may have some or all of the following characteristics: eccentric epithelial atrophy with hair shafts in close proximity to the dermis; concentric lamellar fibroplasia; variably dense perifollicular inflammation, infundibular fusion, total destruction of the follicular epithelium

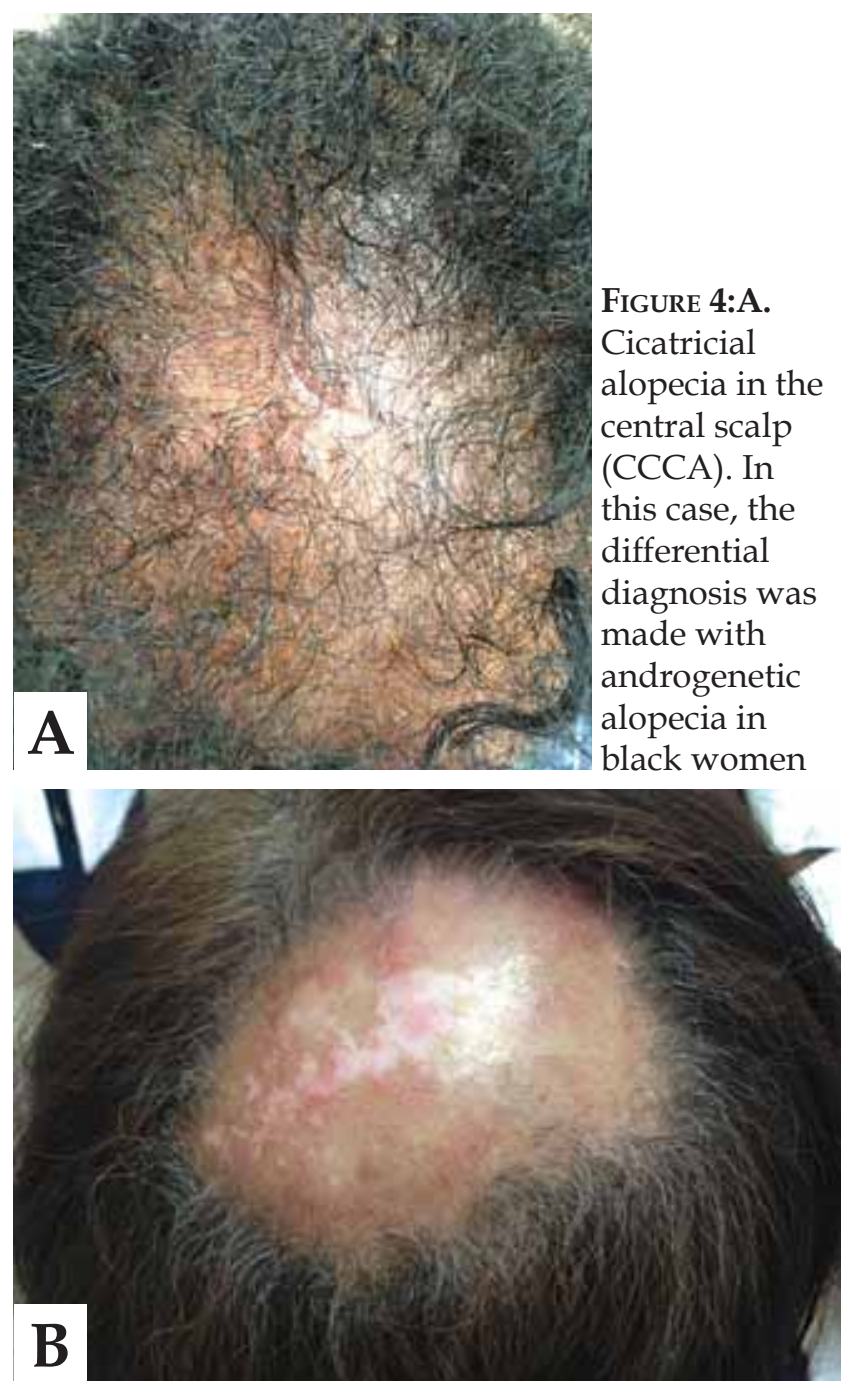

B. LED of the scalp. Because it shows central involvement, it is important to remember the differential diagnosis with CCCA in melanodermic patients with retained hair shaft fragments, granulomatous inflammation (in advanced lesions) and intrafollicular and perifollicular infiltrates rich in neutrophils, and lymphocytes in pustular lesions. ${ }^{37}$

Dermoscopy shows the same findings that are found in other types of scarring alopecia, with absence of follicular ostia, presence of some vellus hairs and terminal hairs of irregular distribution within the area of alopecia, erythema and scaling on the periphery of the plaque, indicating disease activity. ${ }^{32,38}$

The differential diagnosis of CCCA should be made with androgenetic alopecia, alopecia areata, traction alopecia, lupus and Brocq's pseudopelade (Figure 4). ${ }^{4,22}$ The absence of follicular ostia distinguishes CCCA from other non-cicatricial alopecia with similar morphology. Brocq's pseudopelade may represent a distinct form or final stage of CCCA. It presents as asymptomatic macules or plaques in the parietal region, which converge forming an irregular, polycyclic and atrophic area. The presence of pustules, crusts and inflammation in alopecia zones should make the physician suspect the diagnosis or think about a superinfection by fungi and bacteria. ${ }^{32}$

To date, treatment recommendations are based on anecdotes or case reports. The main goal is to stop disease progression, especially in peripheral areas, and relieve symptoms by administering anti-inflammatory agents. Medium- or high-potency intralesional and topical corticosteroids are the first-line treatments. ${ }^{22,32}$

Intralesionalcorticosteroidssuchastriamcinolone $(2.5-5 \mathrm{mg} / \mathrm{ml})$ are administered monthly for a period of at least 6 months, and then symptomatically. Topical corticosteroids are administered daily until stabilization of the clinical condition, and then three times a week for maintenance. In cases in which the use of corticosteroids shows little or no improvement, the administration of topical tacrolimus $(0.1 \%$ ointment twice a day) may be tried. ${ }^{30,32}$

In severe cases or cases with much inflammation, the combination of oral tetracycline (e.g. doxycycline or minocycline) with topical corticosteroids generally shows satisfactory results. Other second-line drugs include: anti-malarials such as hydroxychloroquine (400 mg daily), cyclosporine and mycophenolate mofetil, mainly used in patients with active, recalcitrant disease. ${ }^{32}$

Topical minoxidil $2 \%$ or $5 \%$ may be useful for stimulating viable hair follicles, once the inflammation process is controlled. In the advanced stages with irreversible loss of follicles, hair transplantion is an option, but should only be performed if the inflammatory process has been inactive for at least one year. However, the expectations of the patient should be realistic, given that the survival rate of the transplanted graft may decrease due to the presence of 
scarring areas on the scalp. ${ }^{32}$

In addition to drug treatment, potentially harmful practices to the hair, such as chemical treatments, thermal damage, traction, caustic cosmetics and the use of hot combs should be avoided. These are behavioral measures which constitute an important basis for therapeutic success. ${ }^{4,22}$

\section{d.2) Lichen Planus Pilaris}

Among the predominantly lymphocytic types of alopecia there are the classic form, lichen planus pilaris (LPP), and its variants: frontal fibrosing alopecia (FFA) and the Graham-Little-Picardi-Lasseur syndrome. ${ }^{35}$

Lichen planus pilaris is an uncommon inflammatory disease characterized by a selfdestructive lymphocytic reaction of the hair follicle causing permanent alopecia of the scalp and sometimes the involvement of other areas of the body other than the scalp. ${ }^{39,40}$ Perifollicular erythema, desquamation and keratotic follicular papules are common findings. ${ }^{39}$

According to the North American Hair Research Society classification of cicatricial alopecia, LPP is classified as a primary lymphocytic cicatricial alopecia in which the follicle is self-destroyed with little involvement of the interfollicular dermis. ${ }^{39}$

It occurs more frequently in Caucasians with a male/ female ratio of 1.8 to 1 , and the majority of adult patients are aged 25-70 years. The disease may present alone or in combination with other forms of lichen planus, such as cutaneous, nail or mucosal lichen planus. ${ }^{39}$

To date, there are no data on the prevalence of LPP and its variants in black women. Studies only mention the prevalence of frontal fibrosing alopecia in these patients. ${ }^{41}$

Although the pathogenesis of LPP remains unclear, the most accepted theory is that it is an autoimmune disorder in which activated $\mathrm{T}$ lymphocytes attack the follicle, and this reaction, mediated by cells, is potentially initiated by the action of infectious agents (hepatitis C, HIV, HSV2, HPV, Helicobacter pylori, Treponema pallidum), medical agents (beta blockers, thiazides, antimalarial drugs, ACE inhibitors) and sensitizing agents (gold, mercury and cobalt). These agents bind to keratinocytes and to the follicular epithelium inducing the production of cytokines and chemotactic factors, which trigger the inflammatory response. ${ }^{39}$

The classical LPP lesion is characterized by whitish, atrophic cicatricial areas of the scalp with loss of follicular openings and small islands of residual hairs. Erythematous perifollicular macules, desquamation and keratotic plugs may be observed at the periphery of the alopecia.. ${ }^{39}$ Lesions may be single or multiple, focal or extensive, and more frequently involve parietal areas and the vertex. There maybe also present a reticular pattern that coalesce, reaching large areas (Figure 5). ${ }^{42}$

Clinical features of LPP are hair loss, severe itching, desquamation and burning sensation, which may be aggravated by stress, ultraviolet light and sweating. The stability of this condition is revealed by asymptomatic non-inflamed lesions and negative traction test. At this stage, it may be clinically indistinguishable from other lymphocytic
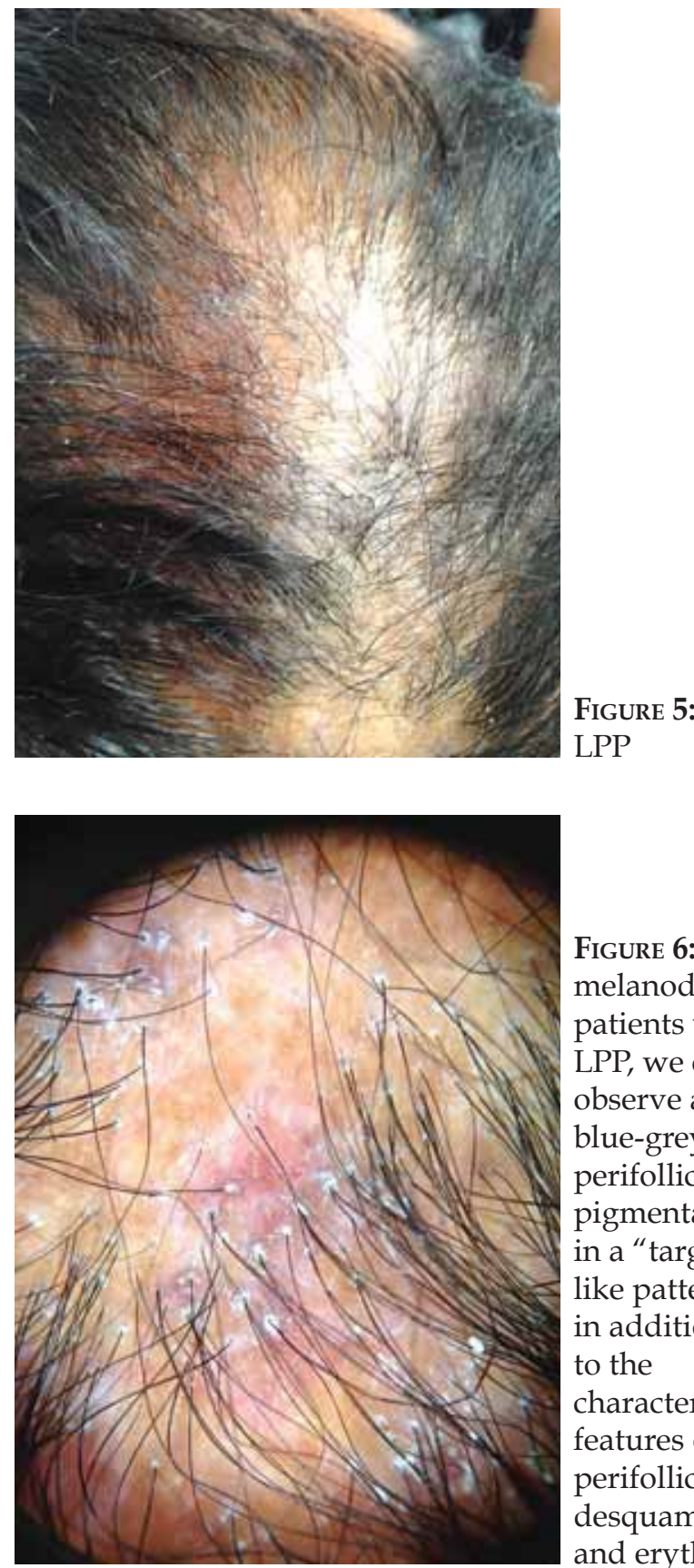

Figure 6: In melanodermic patients with LPP, we can observe a blue-greyish perifollicular pigmentation in a "targetlike pattern", in addition to the characteristic features of perifollicular desquamation and erythema 
inflammatory disorders. ${ }^{39}$ The differential diagnosis should be made with cutaneous lupus erythematosus and other forms of primary lymphocytic cicatricial alopecia. Scleroderma, erythema dyschromicum perstans, dermatitis herpetiformis and hyperthyroidism may be seen in association with LPP. ${ }^{39,41,42}$

Its dermoscopic findings are characterized by: a pigmented network, pinpoint white dots corresponding to the excretory duct of the eccrine gland as well as erythema and perifollicular desquamation in active disease. Gray-bluish perifollicular dots in a target-like pattern may be observed, especially in melanodermic patients (Figure 6).

Histopathological findings are initially characterized by lichenoid lymphocytic infiltrate affecting the infundibulum, isthmus and then the lower region of the follicle. Interface dermatitis occurs between the follicular epithelium and adjacent dermis with loss of the basement membrane zone. Sebaceous glands are lost and the follicular sheath is destroyed. Finally, the structure of the follicle is replaced by lamellar perifollicular fibrosis. Direct immunofluorescence may show colloid bodies with anti-IgM, anti-IgA, anti-IgG and C3 in the dermoepidermal junction or around the infundibulum. ${ }^{43,44}$

Management of LPP begins with an effort to reduce the subjective symptoms, and prevent inflammation and dissemination. Therapeutic strategies depend on the severity of the disease, patient's age and experience of the physician. Although there is no curative treatment modality, multiple treatments have been described, such as intralesional or topical corticosteroids for mild to moderate LPP that affects at least $10 \%$ of the surface of the scalp, with variable response; oral steroids are reserved for patients with rapid evolution of the disease, progressive disease, and severe symptoms; hydroxychloroquine has been used in the treatment of LPP, although its mechanism of action remains unknown. It is likely that it interferes with the antigen presentation and cytokine production. If the signs and symptoms persist after three to six months of treatment with corticosteroids and hydroxychloroquine, immunomodulatory agents such as cyclosporine or mycophenolate mofetil may be used. Third-line drugs for the treatment of difficultto-control LPP with aggressive evolution include retinoids, tetracyclines, griseofulvin, thalidomide, dapsone, topical tacrolimus and minoxidil, although the effectiveness of the latter is still controversial. ${ }^{39}$

\section{d.3) Frontal fibrosing alopecia}

Frontal fibrosing alopecia (FFA) is a form of cicatricial alopecia characterized by progressive recession of the frontotemporal hairline. Most women

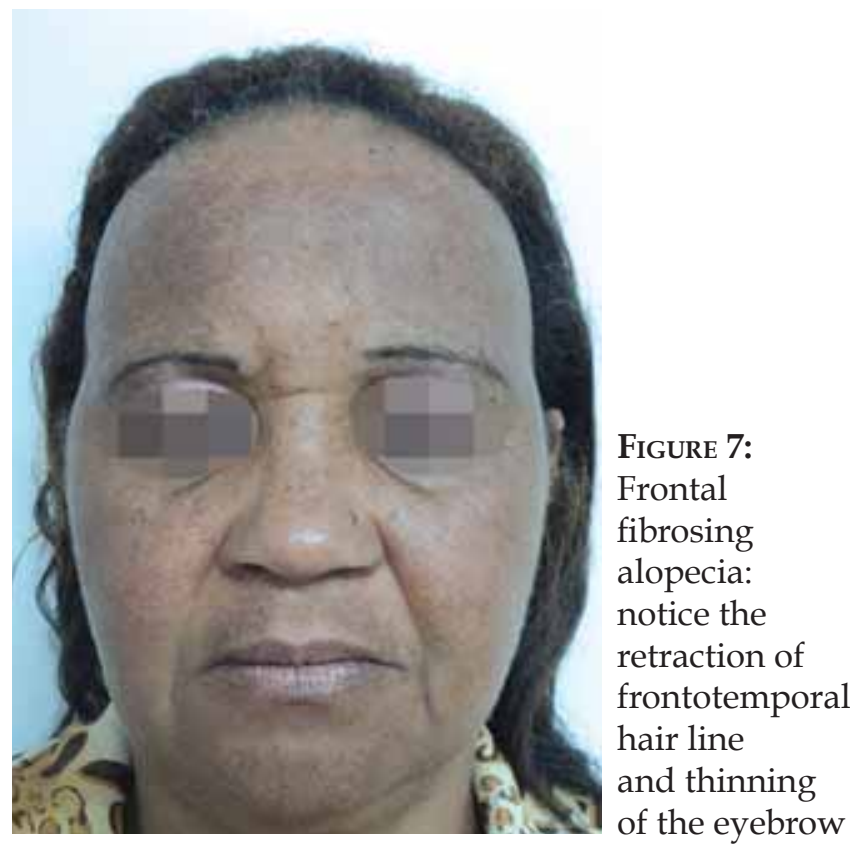

are in the postmenopausal period and there is frequent involvement of the eyebrows and extremities. ${ }^{45,46}$ Hair loss is preceded by perifollicular erythema at the previous hairline and progresses as a symmetrical regression that does not follow the pattern seen in androgenetic alopecia (Figure 7) ${ }^{47}$

The main pathological finding is a follicular destruction mediated by lymphocytes. It belongs, therefore, to the same spectrum of lichen planus pilaris, an autoimmune disorder with specific follicular involvement. ${ }^{48}$

The most important differential diagnosis of FFA in black patients is made with traction alopecia, which affects more than $31.7 \%$ of women in some African communities. Miteva et al first reported the occurrence of FFA in African American women. These authors evaluated 11 black patients ( 10 women and one man), aged 53-72 years, in a study with 141 patients diagnosed with FFA confirmed by biopsy. They found that all black women were postmenopausal and five patients with FFA were associated with signs of traction alopecia. ${ }^{45}$ These patients presented clinically with recent-onset hair loss at the frontotemporal hairline and absence of vellus hairs. Perifollicular desquamation was present around most of the follicles that remained at the hairline. The clue to the diagnosis was provided by the presence of the "lonely hair sign" (Figure 8). It describes the presence of one or two solitary terminal follicles in the middle of the forehead, marking the site of the original hairline, which existed prior to hair loss. Another feature was the alopecia of the eyebrows. Dlova reported the 


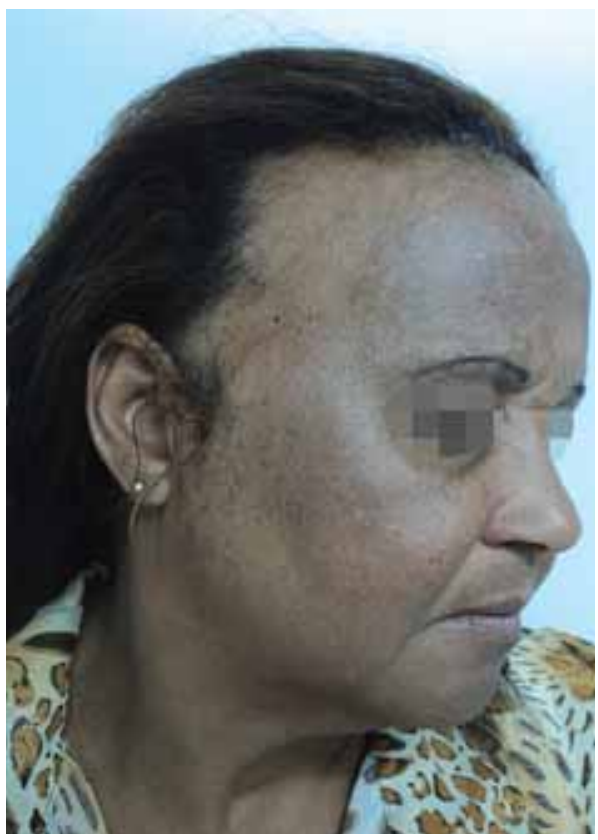

Figure 8: Lonely hair sign: presence of isolated and dispersed terminal hairs in the area of alopecia coexistence of FFA with pigmented lichen planus in 24 black African patients, with an average age of 40 years, which is much lower than the data found in the available literature. According to the author, it could be associated with the mechanical trauma caused by the habits and hairstyles used by black women. This would explain the early manifestation of FFA in African patients. ${ }^{49}$

Hair loss may be sudden, resulting in a rapid regression of the frontal hairline and the loss of the eyebrows, then following a slow and progressive course. In other women, the course of the disease is slow and the disease starts subtly. ${ }^{47}$ The follicles show erythema and mild perifollicular scaling, regarded as evidence of active inflammation. The symmetrical and progressive retraction of the frontal hairline denotes a characteristic pattern which can be easily recognized. ${ }^{47,50}$ The skin in the alopecia area is often pale and smooth, without visible follicular orifices and, in some women, shows a contrast with the adjacent photoaged skin. ${ }^{47}$ In $50-80 \%$ of women a partial or total loss of the eyebrows is observed. This may be an early sign of the disease. There is a trend to greater visualization of blood vessels in the temporal region. Although the main affected areas are the frontal and temporoparietal areas, the marginal follicular loss may rarely extend to the posterior scalp..$^{50}$

The process is usually asymptomatic, but itching may be present. ${ }^{47}$ FFA usually occurs as an independent entity, however, it may also be seen in association with androgenetic alopecia or multifocal lichen planus

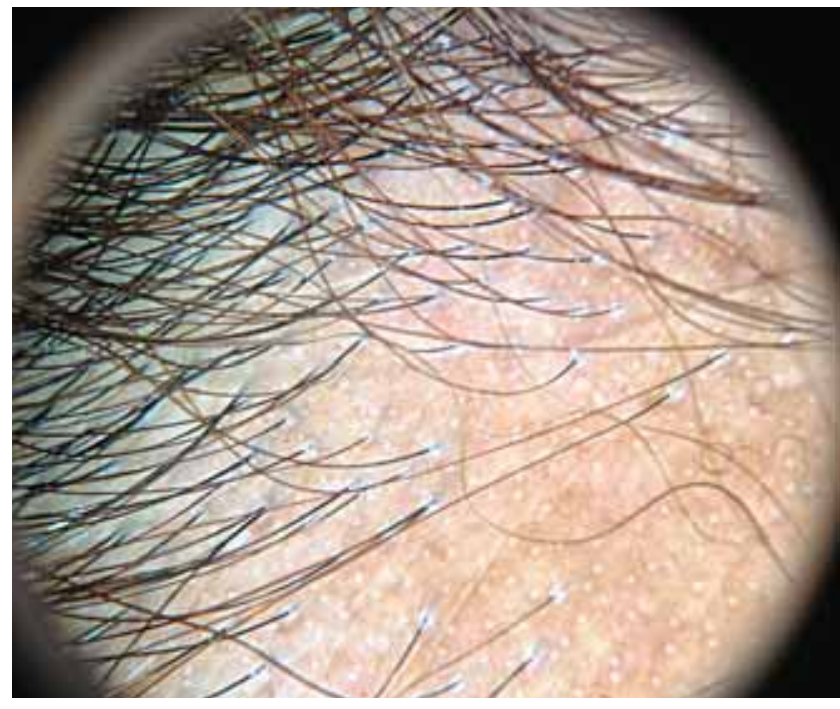

Figure 9: Note the intense perifollicular desquamation which mainly affects the hairs of the frontotemporal region and eyebrows

pilaris. It may be associated with the asymptomatic loss of axillary and pubic hairs, or a more widespread hair loss, particularly on the extremities. This pattern of follicular loss has been associated with the GrahamLittle-Piccardi-Lassueur syndrome, which is primarily characterized by ciatricial alopecia of the scalp, disseminated follicular keratosis and non-scarring hypotrichosis of the axillary and pubic regions. ${ }^{47,51}$

Dermatoscopy of FFA showed the absence of follicular openings and the presence of white dots, scales adhered to the base of the hair shaft, perifollicular erythema, and absence of vellus hairs (Figure 9). ${ }^{35,47}$

Histopathological diagnosis is made by observing the loss of hair follicles, which are replaced by a follicular scar, lichenoid inflammation affecting the follicle in the upper and lower level, and a zone of perifollicular fibrosis, as well as the absence of sebaceous glands. ${ }^{35,47,52}$ The areas that are affected by the lymphocytic inflammation are particularly present outside the concentric fibrosis ring, but are also seen near the follicular sheath, resulting in keratinocyte apoptosis and eventual follicular destruction. ${ }^{47,48,50}$ The immunophenotypes of lymphocytes that reach the follicles show a mixture of CD4- and CD8-activated T cells, similar to those found in lichen planus pilaris. ${ }^{53}$

During the acute phase of hair loss, oral steroids may be indicated, but most women have slowprogression FFA. Other treatment options include topical and intralesional corticosteroids, tacrolimus and pimecrolimus, minoxidil, hydroxychloroquine 
and oral retinoids, but none of them shows consistent results. Finasteride and antiandrogens may be useful, particularly in women who are carriers of concomitant female androgenetic alopecia. ${ }^{47}$

Although it has a very characteristic clinical presentation, FFA in black patients is underdiagnosed. When examining a female patient in the postmenopausal age group who presents a smooth, symmetrical and atrophic area, linear frontotemporal hairline recession with enlargement of the forehead, accompanied by alopecia of the eyebrows, the diagnosis of FFA should be suspected. The differential diagnosis should be made with traction alopecia and ophiasic alopecia areata.

\section{a. Androgenetic alopecia in black women}

Black women naturally have lower follicular density, lower hair growth rate on the scalp and thinner hairs, as described previously. The main complaint of women with androgenetic alopecia is the thinning, and diffuse and progressive shortening of the hairs, which begins in the central parietal area (female alopecia with a pattern distribution), sparing the frontal hairline and giving an impression of increased spread on central hair line..$^{32-35}$

A peculiar characteristic of the black race is the development of "acquired kinky hair". Clinically, it is characterized by the replacement of terminals hairs of the scalp by short, crooked hair, similar to secondary sexual hairs. It usually appears during adolescence, and it is believed that its etiology is influenced by androgens. (Figure 10). ${ }^{36,37,38,54}$

With regard to other etiological, pathophysiological, diagnostic and therapeutic aspects, there is no distinction between the classically described findings of androgenetic alopecia in white women. However, in black women, the differential

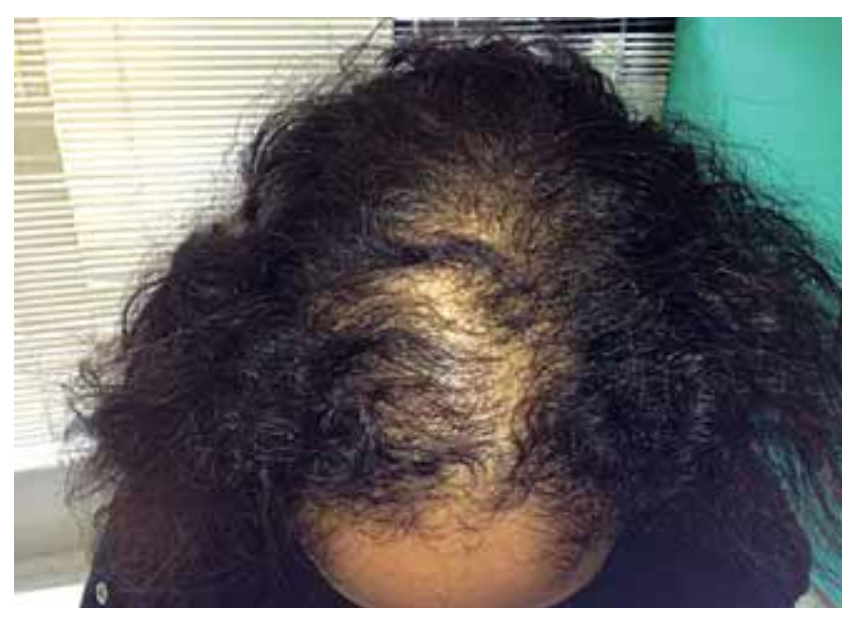

Figure 10: Androgenetic alopecia diagnosis typically related to this race should be remembered, especially if the patient has a long history of hair treatments.

Their dermatoscopic findings will be similar to those found in Caucasian women: more than twenty percent variation in the diameter of the hair shaft, increase in the number of follicular units with only one shaft or focal atrichia, in addition to the evidence of a pigmented network on the sun-exposed scalp.

\section{HAIR STRAIGHENINGANDREAXNG}

HAIR STRAIGHTENING It consists in the temporarily or permanent breakage of chemical bonds of the keratin molecule. Keratin has strong (dissulfide bonds) and weak bonds (hydrogen bridges, van der Waals forces and ionic bonds) that serve to maintain the coiled shape of the molecule. The disruption of disulfide bridges is the mechanism of action of chemical straighteners, and constitutes a permanent phenomenon. The break of hydrolises bridges occurs when the hair is wet and this promotes a temporary hair straightening effect. ${ }^{55}$

\section{TEMPORARY HAIR STRAIGHTENING} METHODS break the hydrogen bridges, and this process is called keratin hydrolysis. The "hot comb", a widely used technique for straightening Afro-ethnic hair, became popular in the early nineteenth century. In this period, many women used a heated metal comb at a temperature of $150-260^{\circ} \mathrm{C}$ to straighten their hairs. ${ }^{56}$ The washing of the hair allows the temporary rearrangement of the hydrogen bridges. The combined use of dryers, hot combs and flat irons consolidate this process, causing dehydration, which gives a new flat structure to the hair shaft and seals the cuticle, making the hair shine, due to a higher reflectivity of light. ${ }^{55}$ The hair must be completely clean and dry before the procedure can be started. Next, vaseline or an oily mixture is applied all over the hair to make it more pliable. During this process, the hot comb combs the full extent of the hair, from the base of hair shaft to the tips of the hair bundles. One should take great care that the comb does not touch the scalp, ears and neck, in order to prevent burns, which are common side effects. ${ }^{10}$ The exposure of hair to humidity, water, shampoos and conditioners reverse the process, and the hair regains its natural look, since this type of hair straightening methods is only temporary ${ }^{55}$

Another common side effect of hot comb is the bubble hair defect. When the hot comb is passed along the length of the hair shaft at very high temperatures, it causes the expulsion of water from the hair. It may emerge from the cortex and advance until the cuticle in the form of water vapor. When this occurs, actual holes are formed in the cuticle, which becomes fragile and 
more susceptible to breakage. This type of damage is less likely to occur if the procedure is performed by a qualified professional, and the hot comb should never be used more than once a week. Today this technique became less popular because of the growing popularity of other hair straightening techniques. ${ }^{10}$

PERMANENT HAIR STRAIGHTENING METHODS are those methods that promote the breakage of keratin disulfide bridges. The most used products are based on sodium hydroxide, lithium and potassium, guanidine hydroxide, bisulfites and ammonium thioglycolate.

- Sodium hydroxides, lithium and guanidine hydroxide (guanidine carbonate and calcium hydroxide) have the highest straightening potential (high $\mathrm{pH}$ ) and are generally chosen by women with afro-ethnic hair. Sodium hydroxide is the chemical that causes more damage to the hair, followed by guanidine hydroxide. It promotes the disruption of disulfide bridges. This process is called lanthionization and consists in the replacement of one third of the cystine in amino acid compositions by lanthionine. The alkaline $\mathrm{pH}$ of the product allows the opening of the cuticle and penetration of the chemical straightener into the cortex. In order to restore the natural $\mathrm{pH}$ of the hair and redo the saline connections, an acidic neutralizing agent is applied and, thus, the hair assumes a straight shape. These products do not fully restore the cystine bridges, therefore, the hair becomes more brittle. ${ }^{55}$

This technique consists first in applying a petrolatum-based cream on the scalp. Then, the hair is divided into four equal parts and the sodium hydroxide is applied to the hairs with the aid of a comb, which should always move from the base to the tips of the hair. The chemical hair relaxer is left on the hair for twenty minutes. After this period of time, it must be removed with water and the hair should be washed with a neutralizing shampoo, which is left on the hair for about five minutes and stops the chemical reaction. The hair should be rinsed again thoroughly to completely remove the neutralizing shampoo. A conditioner is applied to the hair for about ten minutes in order to restore the moisture to the hair, seal the cuticles, soften the hair, prevent knots and also make the hair more malleable and manageable. The hair is rinsed, partially dried and is then ready to be styled. ${ }^{10}$

Straightened or relaxed hair must be washed at least once a week. Ideally, it should be washed twice a week. For protection against daily aggressions, silicone-based products can be used. These products also give more shine to the hair. ${ }^{10}$

- Ammonium thioglycolate or ethanolamine thioglycolate (association between thioglycolic acid and ammonium hydroxide) is an expensive chemical straightener. It is not so strong as those chemicals of the class of hydroxides and it is the chemical most used by Brazilian women. However, it is preferrably used to straighten Caucasian hair, although it may also be used for permanent hair curling/waving. Ammonium thioglycolate is an alkaline agent that promotes swelling of the cuticle. Through this mechanism, it becomes capable of penetrating into the hair cortex and promoting the rearrangement of disulfide bridges. ${ }^{12}$ At this moment, the keratin becomes pliable and can be shaped according to the wishes of the patient: it can be curled with "curlers" (permanent hair curling) or straightened (Caucasian hair), giving a new shape to the hairs. Next, the thioglycolate should be neutralized with an oxidative agent, which generally contains hydrogen peroxide or potassium bromide. The use of this product may cause damage to the hair shaft, cuticle irritation, scalp inflammation and make the hair much more fragile to trauma. ${ }^{55}$

\section{FORMALDEHMDE}

Formaldehyde is one of the most common organic chemicals currently used, because of its low cost and high purity. It is involved in the production of disinfectants, paints, resins, pesticides, among others. As an ingredient in home-made and professional hair straightening products, it has been widely used by the African ethnic population. Its use as a straightening product has been currently prohibited by ANVISA because of its high carcinogenic potential in chronic exposure. It is allowed to be used in the cosmetic industry in concentration of $0.2 \%$ as a preservative and in concentration of $5 \%$ as nail hardener. ${ }^{55}$

The $37 \%$ formaldehyde solution is empirically mixed with liquid keratin, which consists of positively charged amino acids, and with hair conditioning cream. The final product is applied with a comb and the process is finalized with the aid of a hair dryer and a hair straightening iron. Formaldehyde bonds to the proteins of the cuticle and to the hydrolyzed amino acids of the keratin solution to form a hardening layer along the hairs, which makes them waterproof and keeps them hard and straight. Nevertheless, this layer is does not truly protects the hair, because the hair shaft remains fragile and prone to fracture from minor traumas, such as the use of combs and clips. ${ }^{55}$

The restriction on the use of formaldehyde is due to its carcinogenic effect, especially after its volatilization at high temperatures, such as when it is concomitantly used with flat irons and hair dryers devices associated with the hair straightening process. Therefore, its use becomes a risk to the professional and to the user. The chronic inhalation of this chemical is associated with an increased incidence of hematological neoplasms, neoplasms of the central 
nervous system and respiratory tract neoplasms. Acute poisoning is responsible for lesions in the respiratory system, skin necrosis, eye irritation, and may be fatal in high concentrations. ${ }^{55,57}$

It is important to remember that even products labeled "formaldehyde-free" may contain compounds that degrade into formaldehyde when subjected to high temperatures (hair dryers or hair-straightening irons), such as methylene glycol and glyoxylic acid, which are called formaldehyde-releasing chemicals. ${ }^{58}$

Glutaraldehydehasbeenusedashairstraightener ever since the prohibition of formaldehyde. It can be used as a hospital disinfectant at a concentration of $2 \%$ and as a preservative for cosmetic products at a concentration of $0.2 \%$. However, it is prohibited at any concentration for use as a hair straightening product. The inhalation of glutaraldehyde causes even greater damage to the upper respiratory tract than the inhalation of formaldehyde, because it has a ten times higher capacity of generating tissue damage and is potentially carcinogenic. ${ }^{59}$

Currently, formaldehyde-free hair straighteners have glyoxylic acid or methylene glycol in their composition. When in contact with water or heated by hair dryers and flat irons these products release formaldehyde. Formaldehyde then bonds itself the keratin molecule in a cross-linking connection, which allows the alignment of the keratin molecules in pairs (unpublished data). The alignment of keratin molecules gives the hair maximum shine and a maximum straightening effect. Despite the interesting visual aesthetic effect, the steam liberated during the process may cause mutagenicity in the long term.

\section{Henê}

Henê is a popular product in Brazil, used for straightening and coloring the hair. It has a high sales rate and is mainly consumed by young women of African descent. It may be purchased at any drugstore or cosmetic store, since it is not a product developed for professional use, but rather meant for retail sale.

Most products contain pyrogallic acid $\left(\mathrm{C}_{6} \mathrm{H}_{3}(\mathrm{OH})_{3}\right)$ in its chemical composition. Pyrogallic acid is a phenolic compound of organic synthetic origin which may also be called pyrogallol, 1, 2, 3-trihydroxybenzene or 1,2,3-benzenetriol. ANVISA allows the use of pyrogallic acid up to a maximum concentration of $5 \%$, and classifies this product as a coloring agent that acts by oxidizing the hairs. It is not included in the list of hair straightening compounds allowed by ANVISA. It has been currently investigated due to its possible mutagenic and carcinogenic effects. ${ }^{60}$

It is believed that henê may contain sodium hydroxide or lithium hydroxide in its composition, but there is currently no information to substantiate the presence of this active in all product presentations.

Henê can be found in pharmacies in the form of colorless or colored powder or gel, and it has a characteristic pungent odor. Both variants contain pyrogallic acid in their chemical composition. However, the powder variant also contains various plant components, synthetic dyes and corn starch. ${ }^{60}$

Its mode of use varies depending on the manufacturer. However, the product is generally applied with the aid of a comb and is left on the hair for two hours. After this period, the hair should be washed with shampoo and the process should be finalized with the use of a hair conditioner. Initially, applications should be made weekly for 6-8 weeks. After this first phase, the henna should be applied on a fortnightly basis and only on the area where the hair grows. It is important to remember that, if the hair is discolored or has been chemically straightened, an interval of at least 30-45 days should be respected, and that, once the henna is applied, procedures such as hair straightening, relaxing and permanent waving should not be performed within 180 days of the last application. ${ }^{60}$

The hair RELAXING process occurs when thioglycolate is used alone, without the use of flat irons. It produces a less dramatic hair straightening. Note that there must be an interval of at least 15 days between the application of thioglycolate and the use of hair dye agents and permanent dyes, and that thioglycolate is not compatible for simultaneous use with agents from the class of hydroxides. There is the risk of hair tonsure. To reduce the damage caused to the hair and to the scalp, it is essential to use a very thick emollient cream during the hair relaxing procedure. ${ }^{55}$

\section{HARDOS}

NATURAL, TWISTS AND DREADLOCKS:

The choice of keeping the hair in its natural state is not only the most economical option, but also is the healthiest option for the hair, because it is kept away from chemicals. However, not all women with African hair are satisfied with the appearance of their hair and some therefore prefer to adopt a different look. Twists are done by separating a hair bundle from the remaining hair, and dividing it into two equal parts. Next, the two parts are twisted and joined together with beeswax or gel. They must be redone every four weeks. In case this time is exceeded, the hair may get permanently tangled together, and is this hairdo is then called dreadlock which can only be removed by cutting the hair. Some authors have already described cases of traction alopecia secondary to the use of dreadlocks. Although it is a method that used no chemicals, it may also cause alopecia. ${ }^{12}$ 
HAIR BRAIDING: The use of braids is popular among women with African hair. It is often seen as an expression or representation of their culture and personal styles. Moreover, it requires little maintenance, as the braids can be kept for up to three months and the hair must be washed fortnightly or weekly. Moreover, it is an alternative to chemicals used for hair straightening/relaxing/permanent waving. ${ }^{12}$ When braiding the hair, the hairdresser should make sure that there is enough space between the braids, so that the patient is able to touch his/her scalp. This is essential for the maintenance of the braids, because it allows the proper washing of the hair and scalp, and avoids unpleasant effects such as the accumulation of dirt and the production of a characteristic odor. Therefore, the braids should not be made too close together. The shampoo should be applied between the braids and the scalp should be lightly massaged. A milder shampoo may be applied to the braids. They should then be rinsed with running water and left to dry naturally. The success of this hairstyle will also depend on the degree of tension of the braids. If the braids are too tight, the patient may complain of headache and develop traction alopecia. If they are too loose, the patient may feel uncomfortable, as if the hairstyle was unraveling. The braids will naturally become looser with time, indicating the growth of the hair and the time for the hair to be rebraided. ${ }^{10}$ Synthetic or human hair appliques can be added to the braids or braided to the natural hair in order to give volume to it or increase the hair length. The main problems related to the use of braids include traction folliculitis, traction alopecia and acquired trichorrhexis nodosa, although some authors have also described the occurrence of fatigue, nausea, itching, burning and allergic contact dermatitis as a result of the use of synthetic hair. ${ }^{12,61}$ The braids and extensions may be removed manually or by using a suitable solvent. It is important to remember that the braids or extensions should never be cut, because of the risk of damaging the natural incipient hair. ${ }^{12}$

MEGAHAIR (EXTENSIONS): It consists in the addition of synthetic hair fiber or exogenous human hair with the same color characteristics and hair texture to the natural hairs of the patient, in order to make them longer. In order to insert the megahair, the natural hair must be braided close to the scalp. Then the hairpiece is sewn or glued to the base of the natural hair shaft, which ends up getting hidden by the extension (Figure 11). This method also requires low maintenance and the extensions may be washed, treated and stylized according to the wishes of the patient. However, although the use of megahair

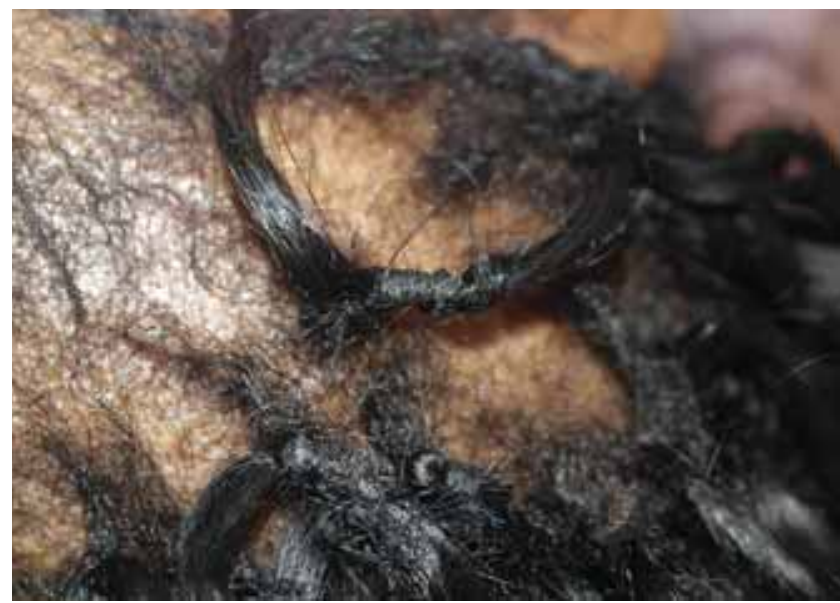

Figure 11: The line used to tie the hairpice, shown in more detail

keeps the natural hairs away from chemicals, traction alopecia, traction folliculitis and contact dermatitis to the glue used to attach the hair extensions have been reported. In these cases, the glue should be removed with great care, using a specific oil. ${ }^{12}$

\section{CONaUSIONS}

Afro-ethnic hair, due to its special characteristic features, is subject to diseases which attack the hair shaft and scalp, and are unique and exclusive to its anatomy. In addition, the hairstyles and hair straightening techniques frequently used by this ethnic group may cause or aggravate cases of alopecia. Brazil is a country with a large number of black and mixed-race people whose hair and hair practices follow the trends and characteristics equivalent to Afro-ethnic hair. It is important to understand and know these peculiarities, because the day-to-day practice of dermatologists requires knowledge of hair and scalp conditions that affect patients with Afroethnic or mixed hair. As important as the prescription of medications is the provision of instructions to these patients about proper hair care. The lack of understanding of the cosmetic needs of this ethnic group by physicians results in the failure to treat most scalp disorders, because patients may not follow the advice of stoping using these practices. When faced with a patient who needs to use other techniques or products, it is important that dermatologists are also able to indicate other cosmetic options for patients with African hair type. 


\section{REFERENCES}

1. Franbourg A, Hallegot $P$, Baltenneck $F$, Toutain $C$, Leroy F.Current research on ethnic hair.J Am Acad Dermatol. 2003;48:S115-9.

2. Alchorne MM, de Abreu MA. Dermatology in black skin. An Bras Dermatol.2008;83:7-20.

3. Burns T, Breathnach S, Cox N, Griffiths C, editors. Rook's Textbook of Dermatology. 8th ed. Oxford: Wiley-Blackwell; 2010.

4. Blume-Peytavi U, Tosti A, Whiting DA, Trüeb RM. Hair Growth and Disorders. Berlin: Springer; 2008. $590 \mathrm{p}$.

5. Miteva M, Tosti A.A detective look' at hair biopsies from African-American patients.Br J Dermatol. 2012;166:1289-94.

6. Sperling LC. Hair density in African Americans. Arch Dermatol. 1999;135:656-8.

7. Ali N. Syed, Anna Kuhajda, Hassan Ayoub, Kaleem Ahmad, Eugene M Frank. African American Hair: Its physical properties and differences relative to Caucasian hair. Cosmetic \& Toilletries. 1995;110;39-48.

8. Whiting DA. The Structure of the Human Hair Follicle: Light Microscopy of Vertical and Horizontal Sections of Scalp Biopsies. New Jersey: Canfield Publishing; 2004. p. 1-31.

9. Somani N, Bergfeld WF. Cicatricial alopecia: classification and histopathology. Dermatol Ther. 2008;21:221-37.

10. Persadsingh N. The hair in black women. Jamaica: Phoenix printery; 2003. 101p.

11. Jennifer M. Fu, MD, and Vera H. Price, MD, FRCP(C). Approach to Hair Loss in Women of Color. Semin Cutan Med Surg. 2009;28:109-14.

12. Callender VD, McMichael A, Cohen GF. Medical and surgical therapies for alopecias in black women. Dermatol Ther. 2004;17:164-76.

13. Callender VD, Wright DR, Davis EC, Sperling LC. Hair Breakage as a presentig signo $f$ early or occult central centrifugal cicatricial alopecia. Arch Dermatol. 2012;148:1047-52.

14. Avashia N, Woolery-Lloyd H, Tosti A, Romanelli P. Short anagen syndrome in na African American woman. J Am Acad Dermatol. 2010;63:1092-3.

15. Kersey PJ. Tricho-dental syndrome: a disorder with a short hair cycle. $\mathrm{Br} \mathrm{J}$ Dermatol. 1987;116:259-63.

16. Van Egmond S, Hoedemaker C, Sinclair R. Short anagen hair nevus: improvement after treatment with 5\% minoxidil. Int J Dermatol. 2007;46:757-9.

17. Samrao A, Price VH, Zedek D, Mirmirani P.The "Fringe Sign" - A useful clinical finding in traction alopecia of the marginal hair line. Dermatol Online J. 2011;17:1.

18. Bolognia JL, Jorizzo JL, Rapini RP. Dermatologia. 2.ed. Rio de Janeiro: Elsevier; 2011.

19. Khumalo NP.The "fringe sign" for public education on traction alopecia. Dermatol Online J. 2012;18:16.

20. Khumalo NP, Jessop S, Gumedze F, Ehrlich R.Determinants of marginal traction alopecia in African girls and woman. J Am Acad Dermatol. 2008;59:432-8.

21. Heath CR, Taylor SC. Alopecia in an ophiasis pattern: traction alopecia versus alopecia areata. Cutis. 2012;89:213-6.

22. Whiting DA, Olsen EA. Central centrifugal alopecia. Dermatol Ther. 2008;21:268-78.

23. Khumalo NP, Jessop S, Gumedze F, Ehrlich R. Determinants of marginal traction alopecia in African girls and women. J Am Acad Dermatol. 2008;59:432-8.

24. Sperling LC. Scarring alopecia and the dermatopathologist. J Cutan Pathol. 2001;28:333-42.

25. Khumalo NP, Ngwanya RM.Traction alopecia: $2 \%$ topical minoxidil shows promisse. Reporto of two cases. J Eur Acad Dermatol Venereol. 2007;21:433-4.

26. Ozçelik D. Extensive traction alopecia attribulate to ponytail hairstyle and its treatment with hair transplantation. Aesthetic Plast Surg. 2005;29:325-7.

27. Rongioletti F, Christana K.Cicatricial (Scarring) Alopecias An Overview of Pathogenesis, Classification, Diagnosis, and Treatment.Am J Clin Dermatol.
2012;13:247-60

28. Moure ER, Romiti R, Machado MC, Valente NY.Primary Cicatricial Alopecias: A Review of Histopathologic Findings in 38 Patients from a Clinical University Hospital in Sao Paulo, Brazil. Clinics (Sao Paulo). 2008;63:747-52.

29. Callender VD, Onwudiwe 0. Prevalence and Etiology of Central Centrifugal Cicatricial Alopecia.Arch Dermatol. 2011;147:972-4.

30. Gathers RC, Lim HW. Central centrifugal cicatricial alopecia: Past, present, and future.J Am Acad Dermatol. 2009;60:660-8.

31. Sperling LC, Sau P. The follicular degeneration syndrome in black patients: 'hot comb alopecia' revisited and revised. Arch Dermatol. 1992;128:68-74.

32. Summers P, Kyei A, Bergfeld W.Central centrifugal cicatricial alopecia - an approach to diagnosis and management.Int J Dermatol. 2011;50:1457-64.

33. Callender VD, Wright DR, Davis EC, Sperling LC. Hair Breakage as a presentig signo $f$ early or occult central centrifugal cicatricial alopecia. Arch Dermatol. 2012;148:1047-52.

34. Kyei A, Bergfeld WF, Piliang M, Summers P. Medical and environmental risk factors for the development of central centrifugal cicatricial alopecia: a population study. Arch Dermatol. 2011;147:909-14.

35. Rongioletti F, Christana K. Cicatricial (Scarring) Alopecias An Overview of Pathogenesis, Classification, Diagnosis, and Treatment.Am J Clin Dermatol. 2012;13:247-60.

36. Somani N, Bergfeld WF. Cicatricial alopecia: Classification and histopathology. Dermatol Ther. 2008;21:221-37.

37. Sperling LC, Cowper SE. The histopathology of primary cicatricial alopecia. Semin Cutan Med Surg. 2006;25:41-50.

38. Ferreira CMM, Barcaui C, Piñero-Maceira J. Atlas de Dermatologia. Aplicação clínica e correlação histopatológica. Rio de Janeiro: Di Livros; 2011. 224 p.

39. Kang H, Alzolibani AA, Otberg N, Shapiro J. Lichen planopilaris.Dermatol Ther. 2008;21:249-56

40. Tan E, Martinka M, Ball N, Shapiro J. Primary cicatricial alopecias: clinicopathology of 112 cases. J Am Acad Dermatol. 2004;50:25-32.

41. Muñoz-Pérez MA, Camacho F. Lichen planopilaris and scleroderma en coup de sabre. J Eur Acad Dermatol Venereol. 2002;16:542-4.

42. Matta M, Kibbi AG, Khattar J, Salman SM, Zaynoun ST. Lichen planopilaris: a clinicopathologic study. J Am Acad Dermatol. 1990;22:594-8.

43. Pai VV, Kikkeri NN, Sori T, Dinesh U. Graham-Little Piccardi Lassueur Syndrome: An Unusual Variant of Follicular Lichen Planus. Int J Trichology. 2011;3:28-30.

44. Trachsler S, Trueb RM. Value of direct immunofluorescence for differential diagnosis of cicatricial alopecia. Dermatology. 2005;211:98-102.

45. Miteva M, Whiting D, Harries M, Bernardes A, Tosti A.Frontal fibrosing alopecia in black patients.Br J Dermatol. 2012;167:208-10.

46. Azulay, DR, Azulay-Abdulafia L. Dermatologia. 5 ed., rev. e atual. reimpr. Rio de Janeiro: Guanabara Koogan; 2011.

47. Dlova NC. Frontal Fibrosing Alopecia and Lichen Planus Pigmentosus: Is there a link? Br J Dermatol. 2013;168:439-42.

48. Trueb RM,Tobin DJ editors. Aging Hair. New York: Springer; 2010. p.33-36.

49. Mirmirani P, Willey A, Headington JT, Stenn K, McCalmont TH, Price VH. Primary cicatricial alopecia:histopatologic findings do not distinguish clinical variants. J Am Acad Dermatol. 2005;52:637-43.

50. Poblet E, Jiménez F, Pascual A, Piqué E.Frontal fibrosing alopecia versus lichen planopilaris: a clinicopathological study, Int J Dermatol. 2006;45:375-80. 
51. Kossard S, Shiell RC. Frontal Fibrosing alopecia developing after hair transplantation for androgenetic alopecia.Int J Dermatol. 2005 Apr;44:321-3.

52. Camacho Martínez F. Frontal fibrosing alopecia: a survey in 16 patients, J Eur Acad Dermatol Venereol. 2005;19:700-5.

53. Kossard S, Lee MS, Wilkinson B.Posmenopausal frontal fibrosing alopecia:a frontal variant of lichen planopilaris.J Am Acad Dermatol. 1997;36:59-66.

54. Boudou P, Reygagne P.Increased scalp skin and serum 5a redutase reduced androgens in a man relevant to the acquired progressive kinky hair disorder and developing androgenética alopecia. Arch Dermatol. 1997;133:1129-33.

55. Abraham LS, Moreira AM, Moura LH, Dias MFRG, Addor FAS. Tratamentos estéticos e cuidados dos cabelos: uma visão médica (parte 2). Surg Cosmet Dermatol. 2009;1:178-85.

56. Abraham LS, Moreira AM, Moura LH, Dias MFRG. Tratamentos estéticos e cuidados dos cabelos: uma visão médica (parte 1). Surg Cosmet Dermatol. 2009;1:130-6.

57. Lewis RJ, Tatken RL, editors. Registry of Toxic Effects of Chemical Substances. On-line Ed. National Institute for Occupational Safety and Health. Cincinnati, $\mathrm{OH}$. LP8925000.March 13, 1989

58. Pierce JS, Abelmann A, Spicer LJ, Adams RE, Glynn ME, Neier K, et al.Characterization of Formaldehyde Exposure Resulting from the Use of Four Professional Hair Straightening Products. J Occup Environ Hyg. 2011;8:686-99.

59. Wickett RR Permanent waving and straightening of hair. Cutis.1987;39:496-7.

60. Rodrigues GB, Stefanello MSB, França AJBDV. Análise das diferenças de composição e modo de aplicação de produtos capilares henê e hena [trabalho de conclusão de curso]. Balneário Camboriú (SC): Universidade do Vale do Itajaí; 2009.

61. Kéïta S, Faye O, Kané BD, Ndiaye HT, Traoré P, Coulibaly K. Hair care and hair styles in women in Bamako, Mali. Int J Dermatol. 2005;44:26-9.

\author{
M AILING ADDRESS: \\ M aria Fernanda Reis Gavazzoni Dias \\ Rua M ariz e Barros 176, 607/608 \\ Icaraí, N iterói. \\ 24220-121. RJ, Brazil. \\ E-mail: alinetanus@hotmail.com
}

How to cite this article: Tanus A, Oliveira CCC, Villarreal DJV, Sanchez FAV, Dias MFRG. Black women's hair: the main scalp dermatoses and aesthetic practices in women of African ethnicity. An Bras Dermatol. 2015;90(4):450-67. 